\title{
Physical Mechanism behind and Access to the I-mode Confinement Regime in Tokamaks
}

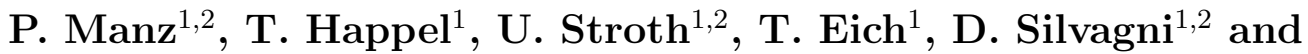 \\ the ASDEX Upgrade Team ${ }^{a}$ \\ ${ }^{1}$ Max-Planck-Institut für Plasmaphysik, Boltzmannstr.2 ,85748 Garching, Germany \\ ${ }^{2}$ Physik-Department E28, Technische Universität München, James-Franck-Str. 1, \\ 85748 Garching, Germany \\ ${ }^{a}$ see the author list of H.Meyer et al. 2019 Nucl.Fusion 59112014
}

\begin{abstract}
The I-mode is an attractive confinement regime for future tokamak based fusion reactors. A model is presented which explains the I-mode regime by the reduction of ITG turbulence near the separatrix at low collisionality, where the separatrix ion temperature can exceed the electron temperature. Drift-Alfvénturbulence develops, with large and small-scale fluctuations being suppressed by phase randomization and finite-Larmor-radius effects, respectively. The intermediate scales form a broad peak in the frequency spectrum, which features the same properties as the characteristic weakly coherent mode. The model, which is studied by means of gyrofluid simulations, reproduces a number of other experimental I-mode observations, such as decoupled energy and particle transport, intermittent turbulent bursts with precursors, an operational window widening with magnetic field strength, and the challenges met when detaching the plasma.
\end{abstract}

\section{Introduction}

In magnetically confined fusion plasmas a transport barrier forms when the plasma heating exceeds a threshold power. Just inside the magnetic separatrix turbulence and the associated radial transport are reduced and steep gradients in density and temperature develop. As a result, plasma density and temperature in the confined plasma rise, which is of critical importance to achieve economical energy production. This operation mode of a tokamak is called the high confinement mode (H-mode), compared to the operation mode without a transport barrier, called the low-confinement mode (L-mode). The H-mode is thought to be maintained by turbulence suppression through pressure gradient driven strong $E \times B$ shear flows. The critical heating power to achieve $\mathrm{H}$-mode is lower when the ion $\nabla B$-drift points towards the active magnetic $\mathrm{X}$-point. When it is directed away from it, the L-H power threshold is higher and another improved confinement regime, called the I-mode [1, 2], can be accessed below the H-mode power threshold. The I-mode shows L-mode like density profiles, but Hmode-like temperature profiles. The I-mode operational space widens with increasing magnetic field strength $[3,4]$. While L-mode confinement is characterized by broadband 
turbulence, the most prominent feature of turbulence in I-mode is the appearance of the so-called weakly coherent mode (WCM) $[5,6,7,8]$. The I-mode is a natural type-I ELM-free regime at improved confinement and does not suffer from strong impurity accumulation making it an attractive confinement regime for future devices, such as DEMO. The reduced particle confinement might have an impact on the fueling requirements.

In this paper the basic idea of a possible mechanism for the decoupling of heat and particle transport is presented (Sec. 2). This mechanism should be strong for rather weak ion temperature gradients or at high ion to electron temperature ratios in the vicinity of the separatrix. The applicability of the underlying assumptions is discussed based on experimental data from the ASDEX Upgrade tokamak (Sec. 3). The proposed mechanism is studied by means of global three-dimensional gyrofluid electromagnetic simulations (Sec. 4). The impact of the ion to electron temperatures ratio and their gradient ratio is presented in Sec. 4.1. The appearance of the weakly coherent mode is reproduced. Decoupling of heat and particle transport is demonstrated in Sec. 4.2. The role of phase randomization at larger scales is discussed in Sec. 4.3. In Sec. 4.4 another experimental feature, enhanced intermittency in the density fluctuations is demonstrated. The impact of steeper electron temperature profiles and flow shear is studied in Sec. 4.5. Implications on I-mode access, in particular the small operational window at small magnetic field strength, are studied in Sec. 5, the runaway to improved confinement and challenges met to reach detachment (Sec. 6), are discussed as well. The paper closes with a summary and the conclusions (Sec. 7).

\section{Turbulence suppression by thermal conduction}

The physical mechanism that selectively reduces only one of the transport channels in I-mode is not understood. In particular a transport barrier induced by a strong $E \times B$ shear flow, as in H-mode, reduces the amplitude in the plasma potential fluctuations [9], hence radial velocity fluctuations are reduced. This suppression mechanism acting on the turbulent amplitude affects all transport channels. In general the dynamics of the density and electron temperature fluctuations in interchange dominated turbulence are identical [10]. Therefore, interchange driven turbulence and in particular ion temperature gradient (ITG) driven turbulence is expected to cause a similar behavior of all transport channels.

A difference in the transport channels can be introduced by the dynamics parallel to the magnetic field. Where the parallel dynamics of electron density fluctuations $\tilde{n}_{e}$ is determined by the parallel velocity only, $\partial_{t} \tilde{n}_{e} \sim-\nabla_{\|} \tilde{u}_{e, \|}[10,11,12]$, electron temperature fluctuations $\tilde{T}_{e}$ are additionally affected by thermal conductivity, $\partial_{t} \tilde{T}_{e} \sim$ $-\nabla_{\|}\left(\tilde{u}_{e, \|}-\tilde{q}_{e, \|}\right)[10,11,12]$. The thermal heat flux $\tilde{q}_{e, \|}$ adds to the parallel dissipation as damping mechanism of temperature fluctuations [10]. At low collisionality, where the parallel dynamics forces the density fluctuations towards the potential fluctuations (called adiabatic response), parallel thermal conductivity levels out the temperature 
fluctuations, which leads to a reduction in heat transport and a steepening of the radial electron temperature gradient. Therefore, simple drift-wave turbulence, which is characterized by reduced electron heat transport with increasing conductivity is a possible regime of turbulence in I-mode.

\section{Ion temperature around the separatrix in the experiment}

To reach such a regime of turbulence a strong interchange drive has to be avoided. The experimentally observed turbulence features in I-mode are the WCM and bursty transport behavior close to the separatrix $\left(0.98<\rho_{\text {pol }}<1.00\right)[7,13,14]$. Therefore, the gradients close to the separatrix are relevant. When the ion $\nabla B$-drift points towards the active magnetic X-point, in the scrape-off layer under attached plasma conditions the temperature ratio is around $\tau_{i}=T_{i} / T_{e}=3[15,16]$. Here we have the case of a sheath connected plasma where the parallel heat transport is dominated by parallel conduction [17] which is higher for electrons than for ions. This leads to a stronger radial decay of the electron temperature. Previous measurements by means of lithium beam charge exchange also showed the ion temperature being at least two times the electron temperature [18]. That the ion temperature at the separatrix can exceed the electron temperatures has also been shown on DIII-D under various conditions $[19,20]$. Figure 1 demonstrates that this is also a reasonable assumption for I-modes in ASDEX Upgrade. The particular discharge shown in Fig. 1 is a deuterium plasma in upper single null configuration with a magnetic field strength of $B=2.5 \mathrm{~T}$ and an edge safety factor of $q_{95}=4$. The plasma has been heated with $\beta_{\text {pol }}$ feedback control as described in detail in Ref. [21] with an average neutral beam power injected of $P_{N B I}=2.3 \mathrm{MW}$ at the time shown in Fig. 1. These are typical temperature profiles in I-mode obtained with this heating scheme. Ion temperatures are measured by charge exchange recombination spectroscopy (CXRS), electron temperatures are obtained from the integrated data analysis IDA [22], relying on electron cyclotron emission (ECE) and Thomson scattering diagnostics. Up to $\rho_{\text {pol }}<0.97$ ion and electron temperatures are indeed similar as reported in Refs. $[23,3,4,21]$, but for $\rho_{\text {pol }}>0.97$ the ion temperature tends to depart from the electron temperature leading to higher ion than electron temperatures at the very edge in agreement with theoretical considerations [17]. In particular the ion temperature measurements exhibit large error bars at the very edge, which makes it difficult to prove that the ion temperature strongly exceeds the electron temperature. However, that ion and electron temperatures have the same values at the separatrix is not supported by the data.

Hence, it appears reasonable to assume that the separatrix conditions are characterized by an ion to electron temperature ratio of $\tau_{i}=2$ and an increased ion temperature gradient scale length $\lambda_{T i}>\lambda_{T e}$. The gradient scale length is $\lambda=-T / \nabla T$. Such conditions will be investigated in more detail using gyrofluid simulations in the following. To the best of our knowledge such a regime has not been studied in literature up to now. In contrast to the case usually considered $\left(\tau_{i}=1, \lambda_{T i}=\lambda_{T e}\right)$, under the 
here presented situation ITG turbulence weakens around the separatrix by two effects: a weaker drive due to the reduced ion temperature gradient and a stabilization of small scale modes by enhanced finite Larmor radius effects [25] due to the increased $\tau_{i}$.

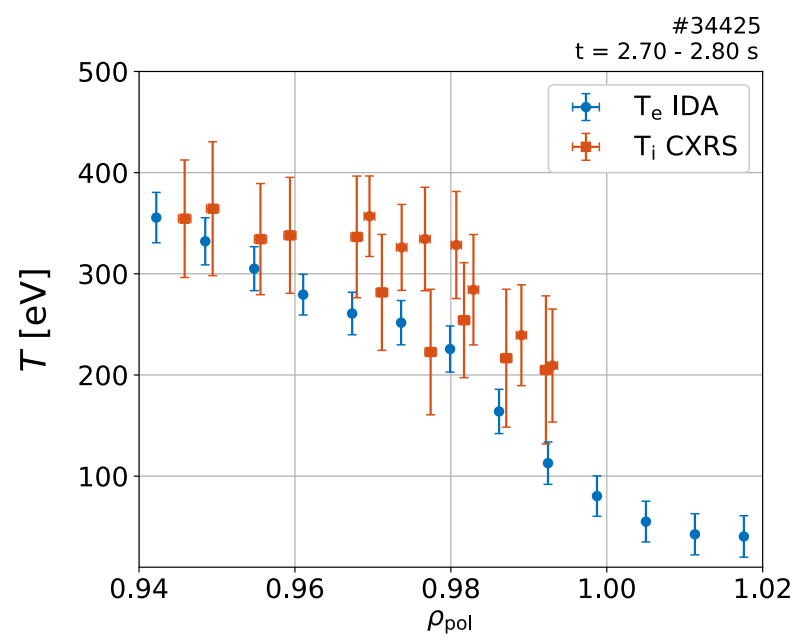

Figure 1. Profiles of electron and ion temperature measured during I-mode in ASDEX Upgrade.

\section{Gyrofluid simulations}

Simulations have been carried out with the global three-dimensional gyrofluid electromagnetic turbulence code GEMR [11, 12]. GEMR solves a six-field model and simulates the densities, parallel velocities, parallel and perpendicular temperatures, and parallel-parallel and perpendicular-parallel heat fluxes for ions and electrons respectively. Plasma potential perturbations including finite Larmor radius corrections are deduced from quasi neutrality. A circular plasma cross-section with toroidal axisymmetry is implemented. The edge of the plasma $(0.96<\rho<1.04)$ is simulated on a $128 \times 512 \times 16$ grid, where $\rho$ is a normalized minor radius. Even though a wider region is simulated, the analysis focuses on the region $0.98<\rho<1.00$ close to the separatrix. GEMR does allow to specify fixed gradient length ratios and the ion to electron temperature ratio $\tau_{i}$ for the entire simulation domain as initial conditions. Therefore, one has to choose the region matching the experimental conditions best. In the cases presented here, the focus is around the last closed flux surface (LCFS). As high $\tau_{i}$ and $\lambda_{T i}>\lambda_{T e}$ applies only for the very edge, the region from mid-pedestal to pedestal top will suffer from unrealistic high $\tau_{i}$.

GEMR is a global model. The kinetic gradients evolve freely, as required by the strength of fluctuating dynamics in the plasma edge region. Transport by gradientdriven turbulence leads to a degradation of the gradients. Profiles are maintained by source/sink zones at the radial boundaries, which are feedback controlled towards the initially specified values [26]. Although GEMR is a global model including a 
time-dependent self-consistent equilibrium, it is based on local model equations. The deviation from the preset background values has to be small. The input parameters are $\delta=\rho_{s} / a$, plasma beta $\beta\left(\bar{n}_{e}, \bar{T}_{e}\right)$, and normalized collisionality $\nu=a \nu_{e} / c_{s}\left(\bar{n}_{e}, \bar{T}_{e}\right)$. Here $\rho_{s}=\sqrt{\bar{T}_{e} m_{i}} / e B$ is the hybrid Larmor radius, $c_{s}=\sqrt{\bar{T}_{e} / m_{i}}$ the cold ion sound speed, $a$ the minor radius, $\bar{T}_{e}$ and $\bar{n}_{e}$ are the reference electron density and temperature, $B$ the magnetic field strength and $\nu_{e}$ the inverse Braginskii collision time. Simulations have been carried out at ASDEX Upgrade parameters (major radius $R=1.65 \mathrm{~m}$, $a=0.5 \mathrm{~m})$. The nominal input parameters at the reference position in the present study are $\delta=1.3 \cdot 10^{-3}, \beta=4.6 \cdot 10^{-6}, \nu=2.4, \tau_{i}=2$ with the reference density $\bar{n}_{e}=1.2 \cdot 10^{19} \mathrm{~m}^{-3}$ and electron temperature $\bar{T}_{e}=120 \mathrm{eV}$. Due to the implemented circular plasma cross-section and the missing terms related to neutral particles, the presented simulations allow for a qualitative investigation. Quantitative deviations from experiments are expected.

\subsection{The impact of ion temperature}

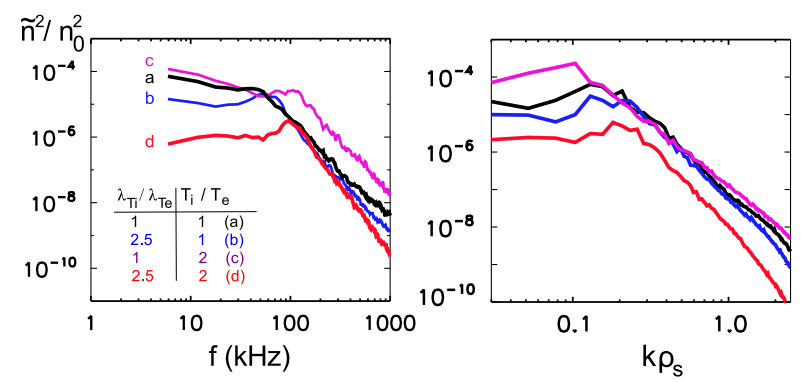

Figure 2. Spectra of density fluctuations for different ion to electron temperature and gradient ratios.

For parameters as usually used in edge simulations $\left(\tau_{i}=1\right.$ and $\left.\lambda_{T i} / \lambda_{T e}=1\right)$ the turbulence shows broadband characteristics in the density fluctuations in frequency space as experimentally observed in L-mode (Fig. 2, case a). Turbulence is ITG driven in this case. Decreasing the ion temperature gradient $\left(\tau_{i}=1, \lambda_{T i} / \lambda_{T e}=2.5\right)$ leads to a reduction in the turbulence amplitude at low and high frequencies and wavenumbers (Fig. 2 case b). The feature of the WCM is now visible (Fig. 2 case b) at frequencies around $f=100 \mathrm{kHz}$ and $k \rho_{s}=0.2$. Increasing the ion temperature instead ( $\tau_{i}=2, \lambda_{T i} / \lambda_{T e}=1$, case c) but keeping the ion temperature gradient steep, ITG turbulence gets even stronger. The turbulence amplitude increases in particular at low wavenumbers. The increase at high frequencies is mainly due to the Doppler shift by increased background $T_{i}$. A very weak WCM is also observed in this case. A WCM can occur also in ITG dominated turbulence. Reducing the ion temperature gradient $\left(\lambda_{T i} / \lambda_{T e}=2.5\right)$ at enhanced $\tau_{i}=2$ leads a strong drop in the turbulence level (Fig. 2, case d) with a prominent WCM. Comparing case d) to case a) the fluctuation level at 
the WCM frequency is roughly the same. In conclusion, an I-mode-like scenario can be obtained by either increasing $\tau_{i}$ or $\lambda_{T i} / \lambda_{T e}$ with respect to $\tau_{i}=1$ and $\lambda_{T i} / \lambda_{T e}=1$.

\subsection{Decoupling heat and particle transport}

To investigate whether this scenario can develop a pedestal in the electron temperature while keeping the density profile similar we will investigate two cases at the same level of particle transport, but different level of heating power in the following.

Figure 3 shows two cases, at different reference temperatures $\left(\bar{T}_{e}=60 \mathrm{eV}\right.$ and $\left.\bar{T}_{e}=100 \mathrm{eV}\right)$. These runs are done at $\tau_{i}=2$ and $\lambda_{T i} / \lambda_{T e}=2$. The total particle flux crossing the last closed flux surface (LCFS) is of the same level, $7.7 \cdot 10^{20} \mathrm{~m}^{-2} \mathrm{~s}^{-1}$ for the case at $\bar{T}_{e}=60 \mathrm{eV}$ and $7.5 \cdot 10^{20} \mathrm{~m}^{-2} \mathrm{~s}^{-1}$ for the case at $\bar{T}_{e}=100 \mathrm{eV}$. The total heat flux crossing the LCFS, which corresponds to the heating power, is $1.6 \mathrm{MW}$ for $\bar{T}_{e}=60$ $\mathrm{eV}$ and 2.7 MW for $\bar{T}_{e}=100 \mathrm{eV}$.

The main difference between both cases is the increased heating power. As seen in the density fluctuation frequency spectra (3c), both cases show the WCM. In the case at lower heating power $\left(\bar{T}_{e}=60 \mathrm{eV}\right)$, the WCM is very weak, and the spectrum is close to broadband L-mode-like turbulence. This case can be considered as an I-mode right after the L-I transition. One may also consider this case as an L-mode just before the L-I transition. The L-I transition is not as sharp as the L-H transition. Increasing the heating power, the density gradient steepens up slightly (Fig. 3a), which is typically observed in the experiment $[3,4,21,23]$. The total particle flux is roughly the same and the electron temperature increases and also steepens up. This qualitatively reproduces the main feature of the I-mode, namely the steepening of the electron temperature gradient without major changes in the density and particle transport. Compared quantitatively to the experiment (for example Fig. 1) the gradients appear less steep. This will be discussed in Sec. 6. As in the experiment, the WCM gets much more pronounced (Fig. 3c) at increased heating power.

For the sake of completeness also the frequency spectra of zonally averaged potential fluctuations are shown. A geodesic acoustic mode (GAM) is clearly observed (Fig. 3d), as in the experiments $[6,7]$. The GAM frequency increases with $T_{e}$. However, it should be noted that I-mode-like cases without GAMs have also been observed in the simulations during this study. These simulations exhibit lower fluctuation levels than the ones presented here. In these simulations the drive of the GAM is weaker. Also the dissipation by Landau damping of the GAM is rather high in these simulations. The edge safety factor is relatively high $\left(q_{s} \approx 5\right)$ and $\tau_{i}=2$.

The main mechanism of reduction in the electron heat transport proposed here (Sec. 2) is the enhanced dissipation by conduction. It can be estimated by the energy exchange between electron temperature fluctuations $\tilde{T}_{e}$ and fluctuations in the parallel electron thermal heat flux $\tilde{q}_{e, \|}$ given by $\left\langle\tilde{T}_{e} \nabla_{\|} \tilde{q}_{e, \|}\right\rangle$. By normalizing to $\frac{1}{2}\left\langle\tilde{T}_{e}^{2}\right\rangle$ one obtains 
a transfer rate

$$
\gamma_{\text {diss }, \text { cond }}=\frac{\left\langle\tilde{T}_{e} \nabla_{\|} \tilde{q}_{e, \|}\right\rangle}{\left\langle\frac{1}{2} \tilde{T}_{e}^{2}\right\rangle} .
$$

The dissipation rate of electron temperature fluctuations by conduction averaged over the edge region $(0.98<\rho<1.00)$ increases from $\gamma_{\text {diss,cond }}=0.8 \mathrm{kHz}$ in the weak I-mode case $\left(\bar{T}_{e}=60 \mathrm{eV}\right)$ to $\gamma_{\text {diss,cond }}=5.5 \mathrm{kHz}$ in the pronounced I-mode case $\left(\bar{T}_{e}=100\right.$ $\mathrm{eV})$. This is an increase by a factor of seven. Turbulence is driven by radial transport $\left\langle\tilde{u}_{x} \tilde{T}_{e}\right\rangle$ and the background gradient $\left\langle\frac{d T_{e}}{d x}\right\rangle$. The reduced amplitude in $\tilde{T}_{e}$ directly leads to reduced transport $\left\langle\tilde{u}_{x} \tilde{T}_{e}\right\rangle$. But reduced transport also leads to steeper gradients $\left\langle\frac{d T_{e}}{d x}\right\rangle$ which in turn increase the growth rate again. Therefore, the enhanced dissipation might be partially attributed to enhanced drive of fluctuations. It will be shown, that this is not the case by comparing the dissipation rate $\gamma_{\text {diss,cond }}$ to an energy input rate $\gamma_{\text {growth,NL }}$. The drive of the electron temperature fluctuations can estimated by $\left\langle\tilde{u}_{x} \tilde{T}_{e}\right\rangle\left\langle\frac{d T_{e}}{d x}\right\rangle$ which divided by $\frac{1}{2}\left\langle\tilde{T}_{e}^{2}\right\rangle$ gives an effective nonlinear growth rate similar to Ref. [24]

$$
\gamma_{\text {growth }, \mathrm{NL}}=\frac{\left\langle\tilde{u}_{x} \tilde{T}_{e}\right\rangle\left\langle\frac{d T_{e}}{d x}\right\rangle}{\left\langle\frac{1}{2} \tilde{T}_{e}^{2}\right\rangle} .
$$

The nonlinear growth rate averaged in the edge region $(0.98<\rho<1.00)$ drops from $\gamma_{\text {growth,NL }}=45.3 \mathrm{kHz}$ in the weak I-mode case $\left(\bar{T}_{e}=60 \mathrm{eV}\right)$ to $\gamma_{\text {growth,NL }}=39.2 \mathrm{kHz}$ in the pronounced I-mode case $\left(\bar{T}_{e}=100 \mathrm{eV}\right)$. Therefore, the reduction of the drive due to the reduced radial transport is stronger than the effect of the steepened electron temperature gradient. This is due to changes in the cross-phase between $\tilde{u}_{x}$ and $\tilde{T}_{e}$, which will be discussed later in this section in detail. The reduction in growth $\gamma_{\text {growth,NL }}$ roughly corresponds to the increase in dissipation by conduction $\gamma_{\text {diss,cond }}$.

The relative fluctuation levels normalized to their gradients are $\hat{n}=$ $\left(\tilde{n} / n_{0}\right) /\left(\rho_{s} / \lambda_{n}\right)=2.3$ and $\hat{T}=\left(\tilde{T}_{e} / T_{e 0}\right) /\left(\rho_{s} / \lambda_{T e}\right)=1.4$ in the case of the weak I-mode $\bar{T}_{e}=60 \mathrm{eV}$. The relative fluctuation level of the electron temperature is lower than that of the density, which is a sign of the increased dissipation of electron temperature fluctuation by thermal conduction. In the pronounced I-mode case $\left(\bar{T}_{e}=100 \mathrm{eV}\right)$ the relative fluctuation levels normalized to their gradients are $\hat{n}=1.2$ and $\hat{T}=0.7$. Also in this case, the relative electron temperature fluctuation level is below the density fluctuation level. Both fluctuation levels are equally suppressed with increasing $\bar{T}_{e}$. A reduction of the density fluctuation level in I-mode is also observed in the experiment [13]. Why both fluctuation levels are reduced even though they are decoupled is discussed in the following in detail.

To discuss the decoupling of heat and particle flux, the cross-phases between potential and density $\alpha_{\tilde{\phi}, \tilde{n}}$ and between potential and electron temperature $\alpha_{\tilde{\phi}, \tilde{T}_{e}}$ are shown in Fig. 5 for the weak and the pronounced I-mode case. For all cases, at scales below the ion Larmor radius $k \rho_{s}>1 / \sqrt{2 \tau_{i}}=0.5\left(k \rho_{i}>1\right.$ with $\left.\rho_{i}=\sqrt{2 \tau_{i}} \rho_{s}\right)$ the crossphase is $\pm \pi$. At a cross-phase of $\pm \pi$ the fluctuations do not induce transport. This effect can be seen as finite Larmor radius (FLR) stabilization [25] of the turbulence. The WCM is present in the range $0.1<k \rho_{s}<0.2$. Thermal conductivity enhances not only 

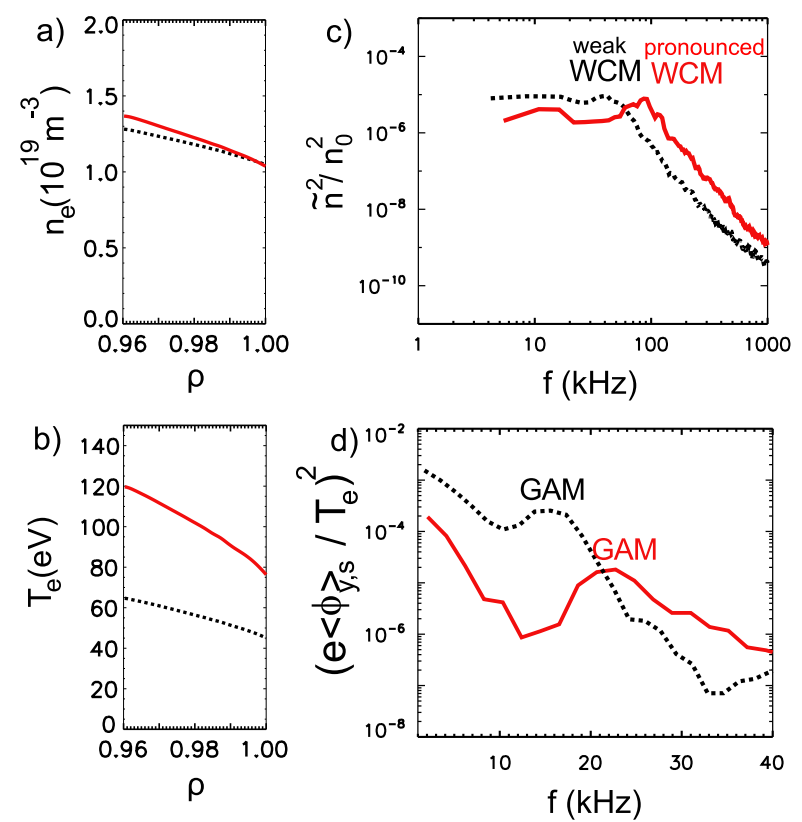

Figure 3. Profiles of density (a) and electron temperature (b) and frequency spectra of density (c) and zonal potential (d) fluctuations for a weak (black dotted lines) and pronounced (red solid lines) I-mode, at a similar level of total particle flux.

the dissipation of the electron temperature fluctuations, but also leads to deviations from an ideal parallel (called adiabatic) response [10]. This means that the difference between the cross-phases $\alpha_{\tilde{\phi}, \tilde{n}}$ and $\alpha_{\tilde{\phi}, \tilde{T}_{e}}$ increases with increasing electron thermal conductivity. At low collisionality, where the parallel dynamics forces the density fluctuations towards the potential fluctuations $\alpha_{\tilde{\phi}, \tilde{n}} \rightarrow 0$, it is expected that the electron temperature exhibits a finite cross-phase to the potential $\alpha_{\tilde{\phi}, \tilde{T}_{e}}>\alpha_{\tilde{\phi}, \tilde{n}}$. Indeed, this is seen in the simulations.

In the weak I-mode case $\left(\bar{T}_{e}=60 \mathrm{eV}\right)$ the turbulence shows interchange characteristics $\alpha_{\tilde{\phi}, \tilde{n}}>\pi / 4$ (Figs. 4e and 5a) and $\alpha_{\tilde{\phi}, \tilde{T}_{e}}>\pi / 4$ (Figs. 4f and 5c), leading to transport, which in turn drives the turbulence. For the more pronounced I-mode $\left(\bar{T}_{e}=100 \mathrm{eV}\right)$ the cross-phase approaches $\alpha_{\tilde{\phi}, \tilde{n}} \rightarrow 0$ (Figs. 4e and 5b), hence the density fluctuations are forced towards the potential fluctuations $\tilde{\phi} \approx \tilde{n}$. This is a characteristic of a drift-wave. However, due to the non-adibaticity induced by electron thermal conductivity $\alpha_{\tilde{\phi}, \tilde{T}_{e}}>\alpha_{\tilde{\phi}, \tilde{n}}$ remains finite (Fig. 5d). The associated perpendicular heat transport drives the WCM.

The enhanced dissipation can be seen in the fluctuation level. Even though the drive of density fluctuations is much lower than that of the electron temperature fluctuations, both fluctuation levels are equally reduced, which can only be ensured by strongly enhanced dissipation of electron temperature fluctuations. This is also the reason why $\hat{n}>\hat{T}$.

The reduction in $\tilde{T}_{e}$ can explain the steepening of the electron temperature gradient. The question arises, why does the density gradient not steepen up, too? This is due to the increasing role of electromagnetic effects. The electrostatic contribution to the 

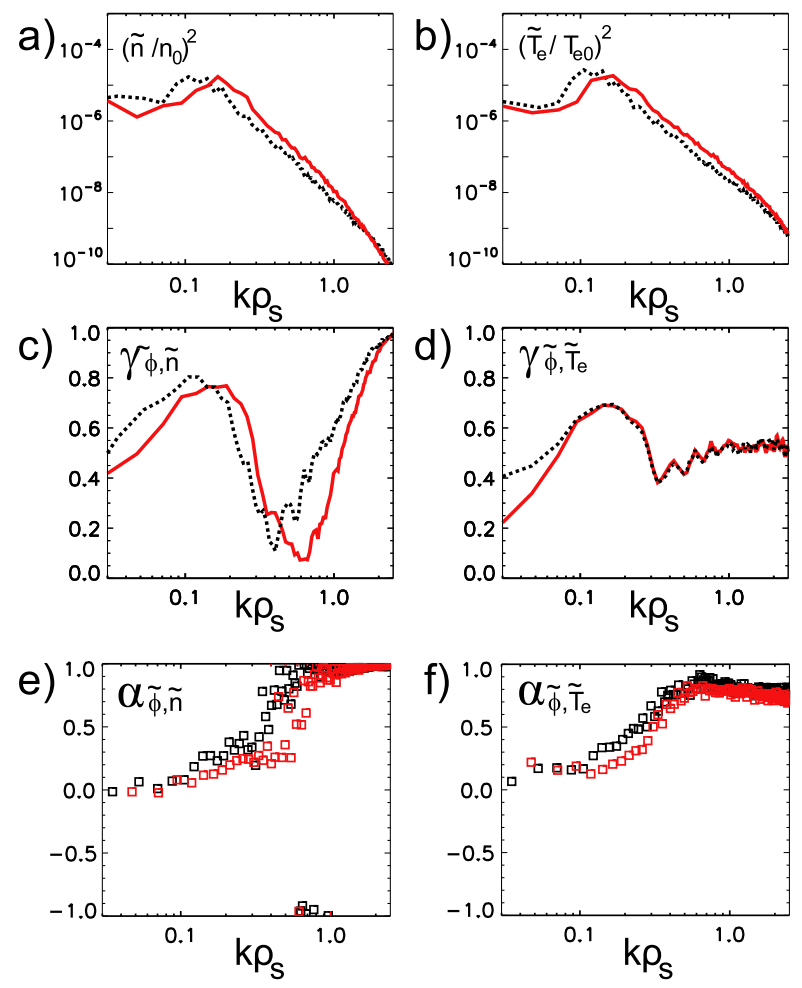

Figure 4. Wavenumber spectra of density (a) and electron temperature fluctuations (b). Cross-coherency between density and potential (c) and electron temperature and potential (d) fluctuations. Cross-phases between density and potential (e) and electron temperature and potential (f) fluctuations. Black dotted (red solid) lines show the weak (pronounced) I-mode case shown in Fig. 3.

particle flux reduces with increasing $\bar{T}_{e}$, the electromagnetic contribution increases from 4 to $33 \%$ of the total particle flux by increasing $\bar{T}_{e}$ from 60 to $100 \mathrm{eV}$. This compensates for the reduction in the electrostatic particle transport. At the same time the relative electromagnetic contribution to the total heat flux increases from $0.1 \%$ to $13.4 \%$. One would expect that the electromagnetic transport increases stronger for the heat than for the particle flux with increasing plasma beta. This is also the case, the electromagnetic particle flux increases by a factor of 7 , the electromagnetic electron heat flux increases by a factor 200. Therefore, electromagnetic effects seem to be important for the I-mode, which will be discussed in the following Sec. 4.3.

\subsection{Reduction of turbulence at large scales}

Next, the reduction of the turbulence level at large scales $k \rho_{s}<0.1$ (see Fig. 4a) is addressed in more detail. An established mechanism to suppress turbulent transport is reducing the cross-coherence by phase randomization [27]. The reduction at lower wavenumbers $\left(k \rho_{s}<0.1\right)$ is accompanied by a significant spread in the cross-phase distribution appearing in the region $k \rho_{s}<0.1$ compared to the region of the WCM $0.1<k \rho_{s}<0.2$, which is much more narrow and thus coherent (Fig. 5). Also 

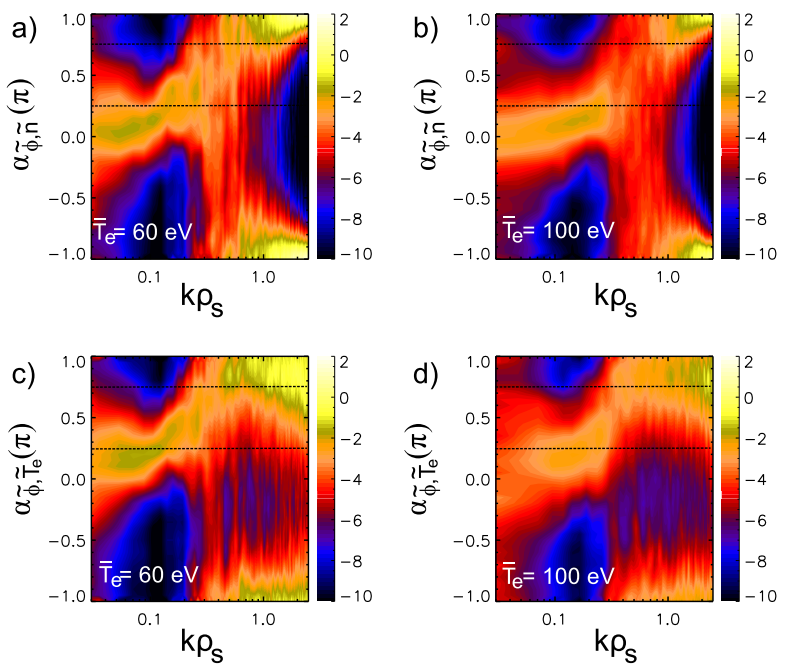

Figure 5. Cross-phase distribution between density $(\mathrm{a}, \mathrm{b})$ or electron temperature $(\mathrm{c}, \mathrm{d})$ and potential fluctuations at different reference temperatures, $\bar{T}_{e}=60 \mathrm{eV}$ (a,c) and $\bar{T}_{e}=100 \mathrm{eV}(\mathrm{b}, \mathrm{d})$. Horizontal dashed lines indicate the boundaries of interchange dominant regions.

the cross-coherency (Figs.4c) and d)) is reduced at larger scales $k \rho_{s}<0.1$. The feature of broadening the phase distribution is called phase randomization. A random distribution of cross-phases does not result in transport, therefore phase randomization is stabilizing. Phase randomization can be induced by field line stochastisation due to magnetic flutter. As magnetic flutter increases with plasma beta, this effect is also called beta stabilization. Tokamak edge turbulence is almost always in the electromagnetic regime as the condition for finite beta effects to be important is scale dependent, $\beta_{e} \geq\left(m_{i} / m_{e}\right)\left(k \rho_{s}\right)^{2}[10,28]$. Therefore, this relation is always fulfilled for sufficiently large structures $\left(k \rho_{s}\right)^{2} \leq \beta_{e} /\left(m_{i} / m_{e}\right)$. In H-mode conditions well above the L-H power threshold it is $\beta_{e} \geq\left(m_{i} / m_{e}\right)$ and electromagnetic effects are important for all scales above $k \rho_{s}<1$. Below this critical beta electromagnetic effects on transport are rather indirect [28]. The electrons become more non-adiabatic by magnetic induction, which also introduces magnetic flutter stabilizing the dynamics [28]. Indeed, with increasing $\bar{T}_{e}$ also electromagnetic effects increase with $\beta_{e}$ and the cross-phase distribution in the region $k \rho_{s}<0.1$ gets less localized, thus more spread and less coherent (Fig. 5). But fluctuations in the magnetic field can lead directly to transport. Thus, phase randomization due to magnetic flutter does not come for free. As discussed above the electromagnetic contribution to particle and heat transport strongly increases from the weak (at $\bar{T}_{e}=60 \mathrm{eV}$ ) to the pronounced I-mode case (at $\bar{T}_{e}=100 \mathrm{eV}$ ). The increased electromagnetic particle transport compensates the reduction in electrostatic particle transport. 


\subsection{Intermittency and density bursts}

During I-mode strongly intermittent turbulent density bursts are observed in the very edge of the confined region $[13,4,29]$. The turbulence amplitude evolution measured by Doppler reflectometry leading up to a burst shows often a wave train (see Fig. 4d in [13]). The temporal separation of the precursor events corresponds to the frequency of the WCM [13]. These features are also reproduced in pronounced I-mode cases simulated here. Figure 6 shows a time trace of the density fluctuation level $\left(\tilde{n} / n_{0}\right)^{2}$ at one point at the outer midplane of the simulation at $\bar{T}_{e}=100 \mathrm{eV}$. The signal is highly intermittent, showing quiet phases and rare strong bursts. Individual bursts sometimes also show precursor fluctuations, where the temporal separation of the precursor events corresponds to the frequency of the WCM. In the GEMR simulations these bursts lead to gentle profile degradation. This phenomenon appeared in a previous study of edge localized modes with GEMR [12]. It is by far not that severe in the simulation presented here. Anyhow, profile degradation leads on long time scales to a departure from the preset background values, which prevent self-consistent simulations on long time scales.

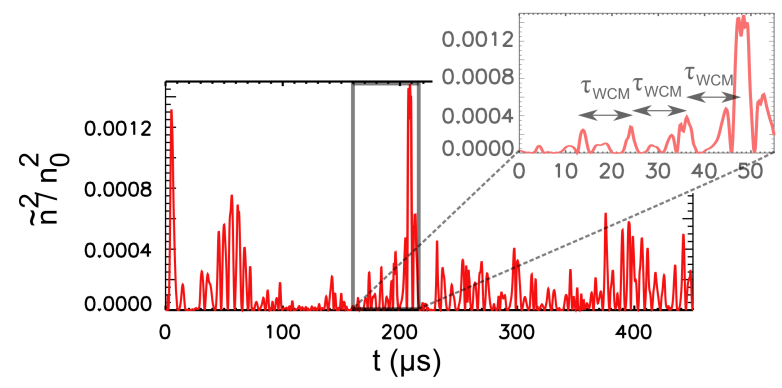

Figure 6. Time trace of density fluctuation level $\left(\tilde{n} / n_{0}\right)^{2}$ at one point at the outer midplane for the pronounced I-mode case at $\bar{T}_{e}=100 \mathrm{eV}$.

\subsection{Impact of steeper electron temperature gradients and flow shear}

During the subsequent evolution in the I-mode towards the H-mode, the electron temperature gradient steepens up and the radial electric field well deepens $[13,4]$. In this section, the evolution of the I-mode is qualitatively imitated by increasing the reference temperature $\bar{T}_{e}$ and decreasing the initial $\lambda_{T e}$ while keeping the reference density $\bar{n}_{e}$ and the initial $\lambda_{n}$ similar. The simulated profiles of four such cases can be seen in Fig. 7i-iii. Compared to the cases studied above (Secs. 4.2-4.4), the profiles are closer to the experimental observation. However, the pedestal top values are still a factor 2 to 3 below the experimental values, which is a significant difference. This will be discussed in more detail later (Sec. 6). By keeping $T_{i}=2 T_{e}$ and $\lambda_{T i} / \lambda_{T e}=2.5$, the ion pressure gradient also steepens up with the increasing electron temperature gradient. With it, the electric field $E_{r} \approx\left(\nabla_{r} p_{i}\right) / e n_{e}$ deepens (Fig. 7iv). This looks qualitatively similar to the experiment $[4,13]$. The case at the lowest reference temperature ((a) in Fig. 7$)$ shows 
broadband turbulence (Fig. 7iv) typical for L-mode. The case (b) shows still broadband turbulence, the case (c) shows the WCM just below $100 \mathrm{kHz}$ (Fig. 7iv). These two cases look like an L-I transition, very similar to the cases studied above (Secs. 4.2-4.4). The fluctuation level increases in L-mode from the case (a) to (b) as the gradients are steeper in case (b). With further increasing temperature gradients the WCM is getting more pronounced (cases (d) and (e) in Fig. 7) and the frequency of the WCM increases. This is also observed in the experiment. Comparing a case deep into L-mode (a) with a case deep into I-mode (d), the turbulence at the WCM frequency can strongly exceed the L-mode turbulence level. At the transition to H-mode, the minimum of the radial electric field is close to $15 \mathrm{kV} / \mathrm{m}$ in ASDEX Upgrade [31]. The cases (d) and (e) are closer to the I-H transition. In experiments in Alcator C-mod the I-H transition is found to be still dominated by shear suppression [32]. Even though both cases exhibit very similar profiles the fluctuation level in the case (e) is strongly reduced compared to case (d). This is due to shear suppression as discussed in the following.
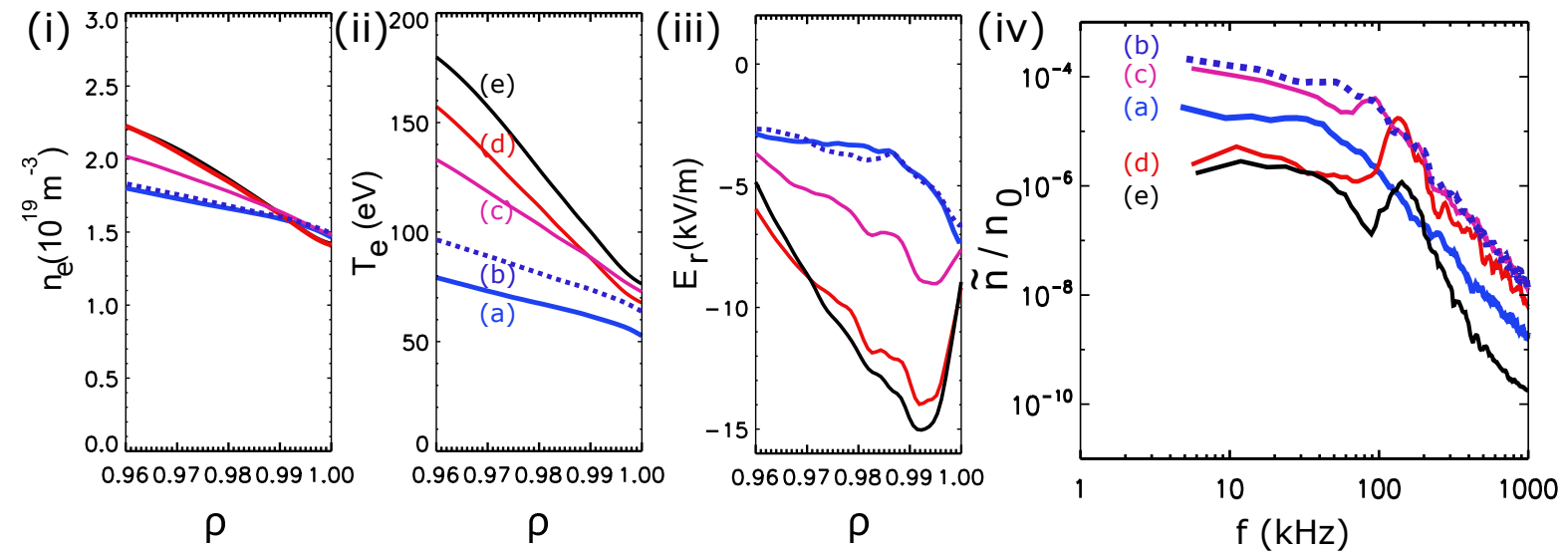

Figure 7. Profiles of density (i), electron temperature (ii) and radial electric field (iii) and frequency spectra of density fluctuations (iv) for increasing electron temperature and its gradient ((a),(c)-(e) in Fig. 8).

Flow shear can suppress turbulence, if the shearing rate $\omega_{s}$ exceeds the eddy-turnover time. Often the eddy-turn over time is approximated by the linear growth rate $\gamma$. Turbulence in the edge of magnetically confined fusion plasmas is characterized by high fluctuation levels. In L-mode, the high fluctuation level, in particular in the small scale-scale vorticity is associated with strong shearing rates, often exceeding the linear growth rates even by orders of magnitudes [33,34]. The small-scale vorticity fluctuations suppress linear instabilities. Instead of being driven by linear instabilities, the turbulence is driven by nonlinear self-sustainment [33, 34]. The nonlinear self-sustained turbulence can be suppressed by the background shear, if the shearing rate exceeds the eddy-turnover time, which is approximately the rms vorticity level $\omega_{s}>\tilde{\Omega}_{\text {rms }}$. Figure 8 shows the vorticity fluctuation level in black and the shearing rate is shown by a dashed red line. In L-mode (case (a)) the vorticity fluctuation level exceeds the shearing rate by an order of magnitude. The shear flow is not strong enough to suppress the turbulence. Also, the 

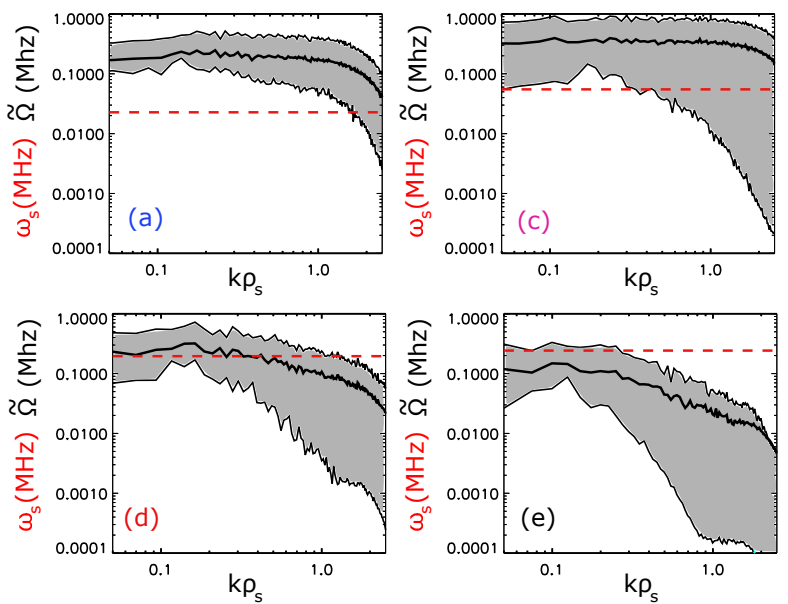

Figure 8. Shearing rate and vorticity for increasing electron temperature and its gradient. The black solid line shows the time average vorticity amplitude. $95 \%$ of the vorticity amplitude distribution in time is below the upper boundary and $50 \%$ of the vorticity amplitude distribution in time is below the lower boundary of the gray region. The lower boundary is the median. The corresponding profiles are shown in Fig. 7.

vorticity is equally distributed across the relevant wavenumber region $0.05<k \rho_{s}<1$. By just enhancing the shear flow all scales would be equally suppressed and it would not lead to the WCM. After the L-I transition (case (c)) the shearing rate is still below the vorticity level on average. Also in this case the shear flow does suppress the turbulence significantly. The reduction in fluctuation level at frequencies smaller and larger the WCM (Fig. 7iv) is not due to the flow shear. The cases (d) and (e) closer to the I-H transition are affected by flow shear. In the case (d) the vorticity level exceeds the shearing rate only at the WCM scale on average. At larges scales the turbulence level is reduced by phase randomization, at small scales by finite Larmor radius stabilization. The reduced vorticity level at these scales allows the flow shear to additionally suppress the turbulence. This makes the WCM much more pronounced in the case (d) compared to the case (c) in Fig. 7iv. In case (e) the flow shear exceeds the vorticity level on average. The average fluctuation level is strongly reduced (Fig. 7iv). Only for a short time the vorticity level can exceed the flow shear making the turbulence appearing much more intermittent as observed in experiment $[4,13]$.

\section{Operational space}

The widening of the I-mode operational window with increasing magnetic field strength can be qualitatively understood by the combined restrictions in beta and collisionality as demonstrated in the following. At sufficiently high collisionality $\left(\nu_{e}^{*}>50\right) T_{i}$ and $T_{e}$ are closely coupled, which is called equipartition [35]. For $\nu_{e}^{*}<10$ ion and electron temperatures can differ substantially $T_{i} / T_{e}>2$ at the separatrix [35] and the ion temperature gradient is weak enough for ITG to not be the dominant drive with 
significant finite Larmor radius stabilization. From the simulations the region in $\beta_{e}$ can be roughly estimated to be within $2 \cdot 10^{-5} \leq \beta_{e} \leq 5 \cdot 10^{-5}$, where the beta stabilization affects structures larger than the WCM. The WCM itself is not stabilized completely and electromagnetic transport is weak.
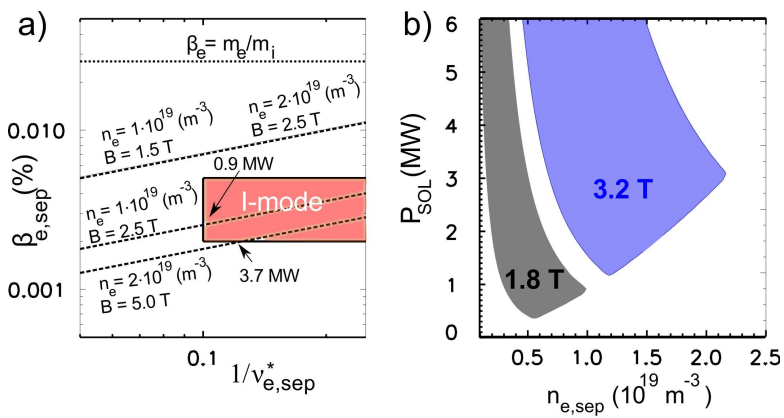

Figure 9. a) Operational window of I-mode in the $\beta_{e}, \nu_{e}^{*-1}$ space. Power scans at different magnetic field strengths and densities are shown by dashed lines. b) Imode access window in power and density space for different magnetic field strengths, $B=1.8 \mathrm{~T}$ in gray and $B=3.2 \mathrm{~T}$ in blue. Scans are at constant safety factor $q_{s}=5$.

This operational window is shown in Fig. 9a by the red box. Several power scans at different densities $n_{\mathrm{e} \text {,sep }}$ and magnetic field strengths have been carried out following the two-point model to calculate the upstream separatrix electron temperature from (Eq. (16.34) in Ref. [36])

$$
T_{\mathrm{e}, \mathrm{sep}}=\left(\frac{1}{\chi_{\perp} n_{\mathrm{e}, \mathrm{sep}} \hat{\kappa}_{e}}\right)^{2 / 9}\left(\frac{7 L_{c} P_{\mathrm{SOL}}}{2 S}\right)^{4 / 9},
$$

with power crossing the separatrix $P_{\mathrm{SOL}}$, perpendicular diffusivity $\chi_{\perp}=1 \mathrm{~m}^{2} \mathrm{~s}^{-1}$, thermal conductivity $\hat{\kappa}_{e}=1.1 \cdot 10^{22}(\mathrm{eV})^{-5 / 2} \mathrm{~m}^{-1} \mathrm{~s}^{-1}$, parallel connection length $L_{c}=$ $\pi q_{s} R$, safety factor $q_{s}=5$, major and minor radii of $R=1.65 \mathrm{~m}$ and $a=0.5 \mathrm{~m}$, respectively, surface area $S=4 \pi^{2} R a \sqrt{\left(1+\kappa^{2}\right) / 2}$ and plasma elongation $\kappa=1.6$. Once the separatrix temperature as function of density and power $T_{\mathrm{e}, \mathrm{sep}}\left(n_{\mathrm{e}, \mathrm{sep}}, P_{\mathrm{SOL}}\right)$ is known the edge collisionality can be calculated $\nu_{e}^{*}\left(n_{\mathrm{e}, \mathrm{sep}}, T_{\mathrm{e}, \mathrm{sep}}\left(n_{\mathrm{e}, \mathrm{sep}}, P_{\mathrm{SOL}}\right)\right)$. The plasma beta $\beta_{\mathrm{e}, \mathrm{sep}}\left(\left(n_{\mathrm{e}, \mathrm{sep}}, T_{\mathrm{e}, \mathrm{sep}}\left(n_{\mathrm{e}, \mathrm{sep}}, P_{\mathrm{SOL}}\right), B\right)\right.$ at the separatrix additionally depends on the magnetic field strength $B$. Therefore, at a given density $n_{\mathrm{e}, \mathrm{sep}}$ and magnetic field strength $B$, edge collisionality $\nu_{\mathrm{e}, \mathrm{sep}}^{*}\left(P_{\mathrm{SOL}}\right)$ and plasma beta $\beta_{\mathrm{e}, \mathrm{sep}}\left(P_{\mathrm{SOL}}\right)$ are functions of the power. In this way the power to reach an operational window in $\beta_{\text {rme,sep }}$ and $\nu_{\mathrm{e}, \text { sep }}$ can be estimated. Examples of such power scans are shown by the dashed lines in Fig. 9a. For $B=2.5 \mathrm{~T}$ and $n_{e}=1 \cdot 10^{19} \mathrm{~m}^{-3}$ I-mode is reached at $0.9 \mathrm{MW}$. For a lower magnetic field $(B=1.5 \mathrm{~T})$ or at a higher density $\left(n_{e}=2 \cdot 10^{19} \mathrm{~m}^{-3}\right)$ the plasma beta overshoots the I-mode operation window and I-mode operation is not possible. At a higher magnetic field $(B=5 \mathrm{~T})$ I-mode operation is again possible at this elevated density, but also higher power is needed to reach I-mode (3.7 MW) compared to the case at lower magnetic field. This can be done for different densities and magnetic field strength. Fig. 9b depicts the regions of I-mode access for two different magnetic fields 
( $B=1.8 \mathrm{~T}$ in gray and $B=3.2 \mathrm{~T}$ in blue). It is assumed that the power is equally distributed between electron and ions and the power is twice the power used to calculate the edge electron temperature. The shape of the L-I power threshold is reminiscent of that of the L-H power threshold and shows an increase at low and high densities. The increase at high densities is rather linear, consistent with previous experimental observations in AUG and Alcator C-mod [3,4]. At higher magnetic fields the access window is much larger and higher edge densities can be reached, for those higher heating powers are necessary, consistent with the experimental findings [3, 4]. Note that the operational space is limited at higher powers by the L-H power threshold, which is not considered in Fig. 9b. To quantitatively compare to the experiments the operational space in $\left(\beta_{\mathrm{e}, \mathrm{sep}}, \nu_{e}^{*}\right)$ should be studied in experiments. From those findings predictions to future devices can be done.

\section{Discussion}

The present simulations are done for limited circular plasmas where gradients can be expected to be less steep than in diverted elongated plasmas. Therefore this study focuses on a qualitative investigations only. The difference in temperature radial gradients between experimental I-mode plasmas and the cases reported in this work is significant. In a $\delta-f$ description the variation of the zonal quantities must be small compared to the reference values $\Delta T_{e} / \bar{T}_{e}<1$. This implies a restriction to the domain size $\Delta r$ as $\Delta T_{e} \approx \Delta r \nabla_{r} T_{e}$. As $L_{T e}=-T_{e} / \nabla_{r} T_{e}$ the domain size should be smaller than the gradient scale length $\delta_{r}<L_{T e}$. To model the evolution of a pedestal extending several gradient scale lengths including the separatrix would require a full-f description. A full-f fluid code including heat conduction such as described in Ref. [37] should allow for more quantitative comparisons to the experiment in the future. Nevertheless $\delta$ - $f$ simulations can help to reveal the fundamental mechanisms causing the phenomenology of the I-mode.

In the following possible explanations of some experimentally observed characteristic features of the I-mode confinement regime are given.

i) The L-I transition: At low temperatures (high collisionality) ions and electrons are closely coupled by equipartition. Their temperature gradients are similar and turbulence is driven by the ITG mechanism resulting in strong broadband particle and heat transport as observed in L-mode. For sufficiently high electron temperatures, ions and electrons can thermally decouple. This leads to the actual transition from L- to I-mode. The main transport reduction from L- to I-mode can be attributed to the stabilization of ITG turbulence by the reduction of its drive, the ion temperature gradient. The GAM appears more prominent closer to the L-I transition than to the $\mathrm{I}-\mathrm{H}$ transition. This is consistent with the experimental observation [32].

ii) The nature of the WCM: In the here presented simulations the WCM is the nonlinear driving region of the turbulence in wavenumber space. The WCM is 
actually the remainder of the broadband turbulence spectrum, where the large scale structures $\left(k<k_{\mathrm{WCM}}\right)$ are suppressed by phase randomization and the small scales $\left(k>k_{\mathrm{WCM}}\right)$ by FLR stabilization. Only just after the L-I transition this is the remainder of the turbulence in L-mode. The turbulence underneath the WCM peak reacts on changes of the background parameters as drift-Alfvén turbulence does. At first (higher collisionality) the WCM still shows interchange characteristics. As the I-mode develops it will transit to more drift-wave characteristics with increasing temperature (decreasing collisionality). By further increasing the temperature (increasing plasma beta) the WCM may feature micro-tearing turbulence (as observed in linear gyrokinetic simulations [38]). The reduction of coherency at larger scales let the WCM appear to be more coherent than the ambient turbulence. The driving range of turbulence is in general more coherent than the ambient turbulence. This is why the WCM can be experimentally observed in coherency preceding the actual L-I transition [21]. Closer to the I-H transition the WCM gets affected by background flow shear.

iii) Runaway effect to improved confinement: The confinement improves with decreasing collisionality and increasing conductivity when the electron temperature increases. This may lead to a runaway effect to improved confinement, finally leading to an I-H transition, which is often observed for I-modes in AUG [23]. The runaway effect can be compensated by reducing the heating power with beta feedback as experimentally demonstrated on AUG [21]. The density fluctuation level is also reduced in I-mode as experimentally observed. The associated reduction in the electrostatic particle flux can be compensated by the electromagnetic particle transport as demonstrated in the simulations.

iv) Detachment: The I-mode regime is difficult to detach, usually an I-L back-transition (not linked to bulk-plasma impurity radiation) is triggered prior to meaningful reductions in parallel heat flux to the divertor [39]. In the present model, the back transition might be explained as follows: If the plasma starts to detach, a large source of cold ions is created in the scrape-off layer from ionization of recycled neutrals from the first wall [16]. As a result $T_{i} / T_{e}$ decreases [16] and the ion temperature gradient steepens up, which is accompanied by stronger particle and heat transport due to resuscitation of ITG turbulence, finally leading to an I-L back-transition.

\section{Summary and Conclusion}

In conclusion, drift-Alfvén turbulence allows for a decoupling of density and electron temperature fluctuations by electron thermal conductivity dissipating electron temperature fluctuations. Gyrofluid simulations with reduced ion temperature gradient and increased ion to electron temperature ratio reproduces several features experimentally observed in the I-mode confinement regime: 
i) The steepening of the electron temperature gradient with increasing heating power without major changes in the density and particle transport (Fig. 3).

ii) The weakly coherent mode appears at frequencies around $100 \mathrm{kHz}$ and $k_{\mathrm{WCM}} \rho_{s} \approx$ 0.1 (Fig. 2) as measured in the experiment [7]. The WCM shows drift-Alfvéninterchange characteristics as found in previous simulation results [30].

iii) Also a geodesic acoustic mode (GAM) appears in the simulations (Fig. 3) as observed in the experiment [7].

iv) The simulated density fluctuations show highly intermittent behavior with strong bursts appearing from time to time (Fig. 6) as observed in the experiment [13]. These bursts also exhibit precursors, connected to the WCM (Fig. 6) as observed in the experiment [13].

v) The reduced operational space at low magnetic fields is consistent with the boundaries of the presented turbulence regime. At moderate plasma beta $\beta_{e}$, high heat conduction can be a blessing by reducing electron temperature fluctuations by $\nabla_{\|} \tilde{q}_{e, \|}$. At high $\beta_{e}$ high heat conduction can turn into a curse by inducing strong radial transport by $\tilde{b}_{r} \partial_{r} \tilde{q}_{e, \|}$. In the simulations large-scale structures are suppressed by phase randomization (Fig. 5), which is a signature of magnetic field line stochastisation. Phase randomization gets stronger with increasing plasma beta $\beta_{e}$ (Fig. 5). This would correspond to an operation window in $\beta_{e}$, since at too high $\beta_{e}$ the electromagnetic transport would be too strong and at too low $\beta_{e}$ phase randomization would be not effective. Furthermore, the edge collisionality $\nu^{*}$ should not be too low allowing for thermally decoupled ions and electrons to avoid a strong ITG drive. A finite operational space in $\beta_{e}$ and $\nu^{*}$ can explain the reduced operational space at low magnetic fields observed experimentally [3, 4] (Fig. 9).

Most of the presented phenomena ask for more detailed studies. Nevertheless, the presented instructive study should guide future experiments and numerical studies using gyrokinetic codes in more realistic geometry.

\section{Acknowledgements}

The authors would to like thank B.D. Scott for providing the code GEMR. This work has been carried out within the framework of the EUROfusion Consortium and has received funding from the Euratom research and training programme 2014-2018 and 2019-2020 under grant agreement No 633053. The views and opinions expressed herein do not necessarily reflect those of the European Commission.

[1] Ryther F. et al. 1998 Plasma Phys. Contr. Fusion 40725

[2] Whyte D. G. et al. 2010 Nucl. Fusion 50105005

[3] Hubbard A. E. et al. 2016 Nucl. Fusion 56086003

[4] Happel T. et al. 2017 Plasma Phys. Contr. Fusion 59014004

[5] Hubbard A. E. et al. 2011 Phys. Plasmas 18056115

[6] Cziegler I. et al. 2013 Phys. Plasmas 20055904

[7] Manz P. et al. 2015 Nucl. Fusion 55083004 
[8] Feng X. et al. 2019 Nucl. Fusion 59096025

[9] Manz P., Ramisch M., Stroth U. 2009 Phys. Rev. Lett. 103165004

[10] Scott B. D. 1997 Plasma Phys. Contr. Fusion 391635

[11] Scott B. D. 2006 Contrib. Plasma Phys. 46714

[12] Kendl A., Scott B. D., Ribeiro T. T. 2010 Phys. Plasmas 17072302

[13] Happel T. et al. 2016 Nucl. Fusion 56064004

[14] Theiler C. et al. 2017 Plasma Phys. Contr. Fusion 59025016

[15] Kočan M. et al. 2012 Plasma Phys. Contr. Fusion 54085009

[16] Carralero D. et al. 2017 Nucl. Fusion 57056044

[17] Stangeby P. C., Leonard, A. W. 2011 Nucl. Fusion 51063001

[18] Reich M. et al. 2004 Plasma Phys. Contr. Fusion 46797

[19] Schaffer M. J. et al. 2001 Phys. Plasmas 82118

[20] Haskey S. R. et al. 2018 Rev. Sci. Instrum. 89 10D110

[21] Happel T. et al. 2017 Nucl. Mat. Energy 18159

[22] Fischer R. et al. 2010 Fusion Sci. Technol. $\mathbf{5 8} 675$

[23] Ryter F. et al. 2017 Nucl. Fusion 57016004

[24] Manz P. et al. 2015 Phys. Plasmas 22022308

[25] Roberts K. V., Taylor J. B. 1962 Phys. Rev. Lett. 8197

[26] Ribeiro T. T., Scott B. D. 2008 Plasma Phys. Contr. Fusion 50055007

[27] Shats M. G., Solomon, W. M., Xia, H. 2003 Phys. Rev. Lett. 90125002

[28] Jenko F., Scott B. D. 1999 Phys. Plasmas 62705

[29] Manz P. et al. 2017 Nucl. Fusion 57086002

[30] Liu Z. X. et al. 2016 Phys. Plasmas 23120703

[31] Sauter P. et al. 2012 Nucl. Fusion 52012001

[32] Czeigler I. et al. 2017 Phys. Rev. Lett. 105105003

[33] Scott B. D. 1992 Phys. Fluids B 42468

[34] Manz P. et al. 2018 Plasma Phys. Contr. Fusion 60085002

[35] Stangeby P. C. 2000 The Plasma Boundary of Magnetic Fusion Devices (Bristol, Institute of Physics (IOP))

[36] Stroth U. 2018 Plasmaphysik - Phänomene, Grundlagen Anwendungen (Berlin, Springer)

[37] Zholobenko W. et al. 2019 Contrib. Plasma Phys. e201900131

[38] Stimmel K. et al. 2019 Phys. Plasmas 26122504

[39] Reinke M. et al. 2019 Nucl. Fusion 59046018 